\title{
The 100 most-cited papers in oral medicine and pathology
}

\author{
Alison José MARTELLI ${ }^{(a)}$ \\ Renato Assis MACHADO(b) \\ Daniella Reis Barbosa MARTELLI(c) \\ Lucimara Teixeira das NEVES(b) \\ Hercílio MARTELLI JUNIOR(c) \\ (a) Universidade Estadual de Campinas - \\ Unicamp, School of Dentistry, Department \\ of Oral Diagnosis, Piracicaba, SP, Brazil. \\ (b)Universidade de São Paulo - USP, Hospital \\ for Rehabilitation of Craniofacial Anomalies, \\ Bauru, SP, Brazil. \\ (c) Universidade Estadual de Montes Claros - \\ Unimontes, Health Sciences Postgraduate \\ Program, Montes Claros, MG, Brazil.
}

Declaration of Interests: The authors certify that they have no commercial or associative interest that represents a conflict of interest in connection with the manuscript.

\section{Corresponding Author:}

Renato Assis Machado

E-mail: renatoassismachado@yahoo.com.br

htpps://doi.org/10.1590/1807-3107bor-2021.vol35.0020

Submitted: July 10, 2020

Accepted for publication: October 22, 2020

Last revision: November 10, 2020
Abstract: This study aimed to analyze the 100 most-cited papers in the field of oral medicine and pathology over time, identifying the areas of more intense research. Papers in journals of oral medicine and pathology were identified using the Web of Science database. The specified research period was between 1900 and 2019. Descriptive statistics was used to analyze the data. Pearson's correlation analysis was used to explore the relationships among Web of Science citations, Dimensions citations, and Altmetric Attention Score. The number of citations of an article in the top 100 most-cited papers published in 1953 or later ranged from 541 to 3623. The papers were published in 47 different journals. The New England Journal of Medicine, American Cancer Society, and Nature Genetics published the most papers. Authors from 18 different countries published papers on head and neck cancer, craniofacial congenital anomalies, and osteonecrosis. Most of the papers were laboratory and descriptive studies. A correlation analysis showed a strong correlation only between Web of Science and Dimensions citations. In sum, although non-specific journals for pathology and oral medicine published the majority of the 100 most-cited papers, this biometric citation study show that head and neck cancer was the issue with the most citations. Together, these results make an important scientific contribution by providing a historical perspective on the research carried out.

Keywords: Oral Medicine; Pathology, Oral; Bibliometrics.

\section{Introduction}

The publication of scientific papers in peer-reviewed journals is the most common method of disseminating scientific research, and, therefore, a vast amount of published literature is easily accessible in different fields and disciplines. It is estimated that the number of scientific publications double every 15-20 years. ${ }^{1}$ However, readers cannot always assess the quality of the publications they read. For the evaluation of papers, bibliometric indicators have been used and one of the most accepted qualitative assessment of scientific publications is the number of citations. ${ }^{2}$ This parameter can help researchers to verify changing research focuses and to detect and acknowledge gaps. ${ }^{1,2}$ Moreover, highly cited papers may promote change in clinical practice and trigger discussions, controversies, and additional research. ${ }^{3}$ 
The first bibliometric study was performed in 1987 to describe important medical advances reported in the Journal of the American Medical Association. ${ }^{4}$ After this study, several bibliometric studies have been carried out to evaluate the most-cited papers in different areas., ${ }^{5,67}$ In dentistry, the subjects of studies in the field of bibliometrics have included dental, oral, and maxillofacial traumatology; ${ }^{8}$ endodontics; ${ }^{9,10,11}$ general dentistry; ${ }_{i}^{12}$ implant dentistry; ${ }_{i}^{13,14,15}$ paediatric dentistry; ${ }_{;}^{16,17}$ periodontics, ${ }^{18}$ and orthodontics. ${ }^{19,20}$ However, a bibliometric study has yet to be carried out for oral medicine and pathology. Thus, the objective of this study was to perform a bibliometric analysis of the 100 mostcited papers in oral medicine and pathology. This study will enable oral medicine professionals, pathologists, students, and researchers in the field of oral medicine and pathology to recognize the most commonly discussed research and clinical issues, analyzing the papers most cited. Consequently, the results of this study can shed light on the efforts of the researchers to evaluate and/or find solutions to oral diseases, and can aid in identify studies that serve as references in the field.

\section{Methodology}

This bibliometric study was carried out to identify and analyze the 100 most-cited papers in the field of oral medicine and pathology. The search was conducted using the Thomson Reuters Web of Science (WOS) citation indexing database (http://www.webofknowledge. com). Specifically, the work was carried out using the "All databases" option, which includes WOS (the main collection), Derwent Innovations Index, KCI - Korean Journal Database, Russian Science Citation Index, and the SciELO Citations Index. Papers published between 1900 and 2019 were included in the analysis, without restrictions on language.

The search strategy used combined terms with the field tag TS (Topic) and the Boolean operator OR in the advanced search tool: TS: (oral pathology) OR TS: (stomatology) OR TS: (oral medicine) OR TS: (oral oncology) OR TS: (oral disease) OR TS: (dental disease) OR TS: (oral) OR TS: (odont) OR TS: (mouth) OR TS: (teeth) OR TS: (tooth) OR TS: (tongue) OR TS: (oral manifestation) OR TS: (dentistry) OR TS: (dentist) OR TS: (dental). The search was performed in all databases on November 19, 2019 and retrieved a total of 2,645,482 papers that were ordered by number of citations. Two authors (AJM and RAM), independently, selected the papers using the abstracts, and the fulltext versions when necessary. Any disagreement over data extraction was resolved through discussion and consensus. The inclusion criterion was that the paper be on a topic related to the field of oral medicine and pathology. Papers not related to the field of oral medicine and pathology, and papers on periodontology, cariology, anatomic variation, and normal condition were excluded. In cases of disagreement between the two reviewers regarding the inclusion of a paper, a third author (HMJ) made the definitive decision.

After the final list was approved, the following information was extracted for each paper: reference information (authors, title, periodical, year, volume, and page), origin of the paper (as defined by the corresponding author), paper subject, study type, the number of citations in WOS, and the number of citations per year. The International Standard Serial Number (ISSN) and year of creation were also examined for each journal. Additionally, the number of citations in the Dimensions database and Altmetric Attention Score (AAS) were obtained from the Dimensions free app (https://www.dimensions.ai).

Descriptive statistics using counts and proportions were used to describe the papers and the journals included in the study. Pearson's correlation analysis was used to examine the relationships between citation counts for individual papers, the AAS, and the Dimensions total citation count. A Pearson's correlation coefficient $(r)<0.3$ was considered poor, 0.3-0.5 low, $0.5-0.7$ moderate, $0.7-0.9$ high, and $>0.9$ very high. $\mathrm{P}$ values $<0.05$ were considered statistically significant.

\section{Results}

The most cited papers in oral medicine and pathology are listed in Table 1, in descending order based on the number of citations found in WOS. Average WOS citation per year, Dimensions, and AAS are also shown. The 100 most-cited papers were published between 1953 and 2015, and the most productive period was after 1990, with 78 papers out of 100 (Figure 1). The papers were published in 47 
Table 1. The 100 most-cited papers in pathology and oral medicine, ordered by number of the citations.

\begin{tabular}{|c|c|c|c|c|c|}
\hline Rank & References & $\begin{array}{l}\text { WOS } \\
\text { citations }\end{array}$ & $\begin{array}{c}\text { WOS } \\
\text { citations/year }\end{array}$ & Dimensions & AAS \\
\hline 1 & $\begin{array}{c}\text { Vitali C, Bombardieri S, Jonsson R, Moutsopoulos HM, Alexander EL, Carsons } \\
\text { SE, Daniels TE, Fox PC, Fox RI, Kassan SS, Pillemer SR, Talal N, Weisman } \\
\text { MH, European Study Group on Classification Criteria for Siögren's Syndrome. } \\
\text { Classification criteria for Siögren's syndrome: a revised version of the European } \\
\text { criteria proposed by the American-European Consensus Group. Ann Rheum Dis. } \\
2002 ; 61(6): 554-8 .\end{array}$ & 3623 & 213.12 & 3500 & 17 \\
\hline 2 & $\begin{array}{c}\text { Ang KK, Harris J, Wheeler R, Weber R, Rosenthal DI, Nguyen-Tân PF, Westra WH, } \\
\text { Chung CH, Jordan RC, Lu C, Kim H, Axelrod R, Silverman CC, Redmond KP, Gillison } \\
\text { ML. Human papillomavirus and survival of patients with oropharyngeal cancer. N Engl } \\
\text { J Med. } 2010 ; 363(1): 24-35 \text {. }\end{array}$ & 3002 & 333.56 & 3300 & 58 \\
\hline 3 & $\begin{array}{c}\text { Silman AJ. Criteria for diagnosis of Behçet's disease. International Study Group for } \\
\text { Behçet's Disease. Lancet. 1990;335(8697):1078-80. }\end{array}$ & 2859 & 98.59 & 2800 & 3 \\
\hline 4 & $\begin{array}{l}\text { Slaughter DP, Southwick HW, Smejkal W. Field cancerization in oral stratified squamous } \\
\text { epithelium - clinical implications of multicentric origin. Cancer. 1953;6(5):963-8. }\end{array}$ & 2283 & 34.59 & 2300 & 32 \\
\hline 5 & $\begin{array}{c}\text { Gillison ML, Koch WM, Capone RB, Spafford M, Westra WH, Wu L, Zahurak } \\
\text { ML, Daniel RW, Viglione M, Symer DE, Shah KV, Sidransky D. Evidence for a causal } \\
\text { association between human papillomavirus and a subset of head and neck cancers. J } \\
\text { Natl Cancer Inst. 2000;92(9):709-20. }\end{array}$ & 1898 & 99.89 & 2000 & 36 \\
\hline 6 & $\begin{array}{l}\text { Pignon JP, Bourhis J, Domenge C, Designé L. Chemotherapy added to locoregional } \\
\text { treatment for head and neck squamous-cell carcinoma: three meta-analyses } \\
\text { of updated individual data. MACH-NC Collaborative Group. Meta-Analysis of } \\
\text { Chemotherapy on Head and Neck Cancer. Lancet. 2000;355(9208):949-55. }\end{array}$ & 1798 & 94.63 & 1900 & 6 \\
\hline 7 & $\begin{array}{c}\text { Chaturvedi AK, Engels EA, Pfeiffer RM, Hernandez BY, Xiao W, Kim E, Jiang } \\
\text { B, Goodman MT, Sibug-Saber M, Cozen W, Liu L, Lynch CF, Wentzensen N, Jordan } \\
\text { RC, Altekruse S, Anderson WF, Rosenberg PS, Gillison ML. Human papillomavirus } \\
\text { and rising oropharyngeal cancer incidence in the United States. J Clin Oncol. } \\
2011 \text {;29(32):4294-301. }\end{array}$ & 1647 & 205.88 & 1900 & 770 \\
\hline 8 & $\begin{array}{l}\text { Fakhry C, Westra WH, Li S, Cmelak A, Ridge JA, Pinto H, Forastiere A, Gillison } \\
\text { ML. Improved survival of patients with human papillomavirus-positive head and } \\
\text { neck squamous cell carcinoma in a prospective clinical trial. J Natl Cancer Inst. } \\
\qquad 2008 ; 100(4): 261-9 .\end{array}$ & 1597 & 145.18 & 1700 & 25 \\
\hline 9 & $\begin{array}{c}\text { D'Souza G, Kreimer AR, Viscidi R, Pawlita M, Fakhry C, Koch WM, Westra } \\
\text { WH, Gillison ML. Case-control study of human papillomavirus and oropharyngeal } \\
\text { cancer. N Engl J Med. 2007;356(19):1944-56. }\end{array}$ & 1588 & 132.33 & 1800 & 155 \\
\hline 10 & $\begin{array}{c}\text { Vokes EE, Weichselbaum RR, Lippman SM, Hong WK. Head and Neck Cancer. N Engl } \\
\text { J Med. 1993; 328:184-194 }\end{array}$ & 1459 & 56.12 & 1300 & 6 \\
\hline 11 & $\begin{array}{l}\text { Vitali C, Bombardieri S, Moutsopoulos HM, Balestrieri G, Bencivelli W, Bernstein } \\
\text { RM, Bierrum KB, Braga S, Coll J, de Vita S, et al. Preliminary criteria for the } \\
\text { classification of Siögren's syndrome. Results of a prospective concerted action } \\
\text { supported by the European Community. Arthritis Rheum. 1993;36(3):340-7. }\end{array}$ & 1424 & 54.77 & 1200 & 3 \\
\hline 12 & $\begin{array}{c}\text { Cheng AL, Hsu CH, Lin JK, Hsu MM, Ho YF, Shen TS, Ko JY, Lin JT, Lin BR, Ming- } \\
\text { Shiang W, Yu HS, Jee SH, Chen GS, Chen TM, Chen CA, Lai MK, Pu YS, Pan } \\
\text { MH, Wang YJ, Tsai CC, Hsieh CY. Phase I clinical trial of curcumin, a chemopreventive } \\
\text { agent, in patients with high-risk or pre-malignant lesions. Anticancer Res. } \\
\text { 2001;21 (4B):2895-900. }\end{array}$ & 1419 & 78.83 & 1300 & 70 \\
\hline 13 & $\begin{array}{l}\text { El-Sayed IH, Huang X, El-Sayed MA. Surface plasmon resonance scattering } \\
\text { and absorption of anti-EGFR antibody conjugated gold nanoparticles in cancer } \\
\text { diagnostics: applications in oral cancer. Nano Lett. 2005;5(5):829-34. }\end{array}$ & 1396 & 99.71 & 1300 & 17 \\
\hline 14 & $\begin{array}{l}\text { Warnakulasuriya S. Global epidemiology of oral and oropharyngeal cancer. Oral } \\
\qquad \text { Oncol. 2009;45(4-5):309-16. }\end{array}$ & 1350 & 135.00 & 1500 & 139 \\
\hline 15 & $\begin{array}{c}\text { Ruggiero SL, Mehrotra B, Rosenberg TJ, Engroff SL. Osteonecrosis of the jaws } \\
\text { associated with the use of bisphosphonates: a review of } 63 \text { cases. J Oral Maxillofac } \\
\text { Surg. } 2004 \text { May;62(5):527-34. }\end{array}$ & 1286 & 257.20 & 1400 & 4 \\
\hline 16 & $\begin{array}{c}\text { Leemans CR, Braakhuis BJ, Brakenhoff RH. The molecular biology of head and neck } \\
\text { cancer. Nat Rev Cancer. } 2011 ; 11(1): 9-22 \text {. }\end{array}$ & 1272 & 159.00 & 1300 & 13 \\
\hline
\end{tabular}


Kreimer AR, Clifford GM, Boyle P, Franceschi S. Human papillomavirus types in head

17 and neck squamous cell carcinomas worldwide: a systematic review. Cancer Epidemiol Biomarkers Prev. 2005; 14(2):467-75.

Blot WJ, McLaughlin JK, Winn DM, Austin DF, Greenberg RS, Preston-Martin

18 S, Bernstein L, Schoenberg JB, Stemhagen A, Fraumeni JF Jr. Smoking and drinking in relation to oral and pharyngeal cancer. Cancer Res. 1988;48(1 1):3282-7.

19 Cancer Genome Atlas Network. Comprehensive genomic characterization of head and neck squamous cell carcinomas. Nature. 2015;517(7536):576-82.

20 Sakane T, Takeno M, Suzuki N, Inaba G. Behçet's disease. N Engl J Med. 1999;341(17):1284-91.

Hong WK, Lippman SM, Itri LM, Karp DD, Lee JS, Byers RM, Schantz SP, Kramer AM, Lotan

$21 \quad R$, Peters $L J$, et al. Prevention of second primary tumors with isotretinoin in squamous-cell carcinoma of the head and neck. N Engl J Med. 1990;323(12):795-801.

Mundlos S, Otto F, Mundlos C, Mulliken JB, Aylsworth AS, Albright S, Lindhout

D, Cole WG, Henn W, Knoll JH, Owen MJ, Mertelsmann R, Zabel BU, Olsen BR.

Mutations involving the transcription factor CBFAl cause cleidocranial dysplasia. Cell. 1997;89(5):773-9.

Lupski JR, de Oca-Luna RM, Slaugenhaupt S, Pentao L, Guzzetta V, Trask

23 BJ, Saucedo-Cardenas O, Barker DF, Killian JM, Garcia CA, Chakravarti A, Pate

PI. DNA duplication associated with Charcot-Marie-Tooth disease type 1A. Cell. $1991 ; 66(2): 219-32$

24 Petersen PE, Bourgeois D, Ogawa H, Estupinan-Day S, Ndiaye C. The global burden of oral diseases and risks to oral health. Bull World Health Organ. 2005;83(9):661-9.

Petersen PE. The World Oral Health Report 2003: continuous improvement of oral

25 health in the 21 st century--the approach of the WHO Global Oral Health Programme. Community Dent Oral Epidemiol. 2003;31 Suppl 1:3-23.

Khosla S, Burr D, Cauley J, Dempster DW, Ebeling PR, Felsenberg D, Gagel RF, Gilsanz

V, Guise T, Koka S, McCauley LK, McGowan J, McKee MD, Mohla S, Pendrys DG, Raisz LG, Ruggiero SL, Shafer DM, Shum L, Silverman SL, Van Poznak CH, Watts N, Woo

SB, Shane E; American Society for Bone and Mineral Research. Bisphosphonateassociated osteonecrosis of the jaw: report of a task force of the American Society for Bone and Mineral Research. J Bone Miner Res. 2007;22(10):1479-91.

Marx RE, Sawatari Y, Fortin M, Broumand V. Bisphosphonate-induced exposed bone (osteonecrosis/osteopetrosis) of the jaws: risk factors, recognition, prevention, and treatment. J Oral Maxillofac Surg. 2005;63(1 1):1567-75.

Satokata I, Maas R. Msx 1 deficient mice exhibit cleft palate and abnormalities of craniofacial and tooth development. Nat Genet. $1994 ; 6(4): 348-56$.

Chaturvedi AK, Engels EA, Anderson WF, Gillison ML. Incidence trends for human

29 papillomavirus-related and -unrelated oral squamous cell carcinomas in the United States. J Clin Oncol. 2008;26(4):612-9.

Loeys BL, Chen J, Neptune ER, Judge DP, Podowski M, Holm T, Meyers J, Leitch CC, Katsanis N, Sharifi N, Xu FL, Myers LA, Spevak PJ, Cameron DE, De Backer J, Hellemans J, Chen

30 Y, Davis EC, Webb CL, Kress W, Coucke P, Rifkin DB, De Paepe AM, Dietz HC. A syndrome of altered cardiovascular, craniofacial, neurocognitive and skeletal development caused by mutations in TGFBR1 or TGFBR2. Nat Genet. 2005;37(3):275-81.

Züchner S, Mersiyanova IV, Muglia M, Bissar-Tadmouri N, Rochelle J, Dadali

EL, Zappia M, Nelis E, Patitucci A, Senderek J, Parman Y, Evgrafov O, Jonghe

PD, Takahashi Y, Tsuji S, Pericak-Vance MA, Quattrone A, Battaloglu E, Polyakov

AV, Timmerman V, Schröder JM, Vance JM. Mutations in the mitochondrial

GTPase mitofusin 2 cause Charcot-Marie-Tooth neuropathy type 2A. Nat Genet. 2004;36(5):449-51.

Henke M, Laszig R, Rübe C, Schäfer U, Haase KD, Schilcher B, Mose S, Beer $K T$, Burger $U$, Dougherty $C$, Frommhold $H$. Erythropoietin to treat head and neck cancer patients with anaemia undergoing radiotherapy: randomised, double-blind, placebo-controlled trial. Lancet. 2003;362(9392):1255-60. mutations in FGF23. Nat Genet. 2000;26(3):345-8.
1258

89.86

1400

27

1237

39.90

1200

8

$\begin{array}{llll}1234 & 308.50 & 1400 \quad 225\end{array}$

$\begin{array}{llll}1225 & 61.25 & 1300 & 5\end{array}$

$\begin{array}{llll}1182 & 40.76 & 1000 & 3\end{array}$

1146

52.09

1200

13

$\begin{array}{llll}1074 & 38.36 & 1000 & 12\end{array}$

$069 \quad 76.36 \quad 1100$

$\begin{array}{llll}1067 & 66.69 & 1300 & 10\end{array}$

$66.69-1300-10$

$\begin{array}{llll}1032 & 86.00 & 1100 & 19\end{array}$

$\begin{array}{llll}1008 & 72.00 & 1100 & 11\end{array}$

$\begin{array}{llll}980 & 39.20 & 945 & 6\end{array}$

$\begin{array}{llll}970 & 88.18 & 1000 & 34\end{array}$

$\begin{array}{llll}969 & 69.21 & 1100 & 11\end{array}$

$\begin{array}{llll}969 & 64.60 & 1100\end{array}$

$\begin{array}{llll}967 & 60.44 & 1000 & 14\end{array}$

$\begin{array}{llll}938 & 49.37 & 1000 & 6\end{array}$




\begin{tabular}{|c|c|c|c|c|c|}
\hline 34 & $\begin{array}{l}\text { Takemura } \mathrm{H} \text {, Hughes AR, Thastrup O, Putney JW Jr. Activation of calcium entry by the } \\
\text { tumor promoter thapsigargin in parotid acinar cells. Evidence that an intracellular } \\
\text { calcium pool and not an inositol phosphate regulates calcium fluxes at the plasma } \\
\text { membrane. J Biol Chem. } 1989 ; 264(21): 12266-71 \text {. }\end{array}$ & 923 & 30.77 & 664 & - \\
\hline 35 & $\begin{array}{l}\text { Marur S, D'Souza G, Westra WH, Forastiere AA. HPV-associated head and neck } \\
\text { cancer: a virus-related cancer epidemic. Lancet Oncol. 2010;1 1(8):781-9. }\end{array}$ & 915 & 101.67 & 1000 & 30 \\
\hline 36 & $\begin{array}{l}\text { Gillison ML, D'Souza G, Westra W, Sugar E, Xiao W, Begum S, Viscidi R. Distinct risk } \\
\text { factor profiles for human papillomavirus type 16-positive and human papillomavirus } \\
\text { type 16-negative head and neck cancers. J Natl Cancer Inst. 2008;100(6):407-20. }\end{array}$ & 911 & 82.82 & 968 & 466 \\
\hline 37 & $\begin{array}{c}\text { Bergoffen J, Scherer SS, Wang S, Scott MO, Bone LJ, Paul DL, Chen K, Lensch } \\
\text { MW, Chance PF, Fischbeck KH. Connexin mutations in X-linked Charcot-Marie-Tooth } \\
\text { disease. Science. 1993;262(5142):2039-42. }\end{array}$ & 902 & 34.69 & 863 & 9 \\
\hline 38 & $\begin{array}{c}\text { Chisholm DM, Mason DK. Labial salivary gland biopsy in Siögren's disease. J Clin } \\
\text { Pathol. 1968;21(5):656-60. }\end{array}$ & 889 & 17.43 & 740 & - \\
\hline 39 & $\begin{array}{l}\text { Woo SB, Hellstein JW, Kalmar JR. Narrative [corrected] review: bisphosphonates and } \\
\text { osteonecrosis of the jaws. Ann Intern Med. 2006;144(10):753-61. }\end{array}$ & 883 & 67.92 & 944 & 15 \\
\hline 40 & $\begin{array}{l}\text { Burkitt D. A sarcoma involving the jaws in African children. Br J Surg. } \\
\qquad 1958 ; 46(197): 218-23 .\end{array}$ & 874 & 14.33 & 919 & 21 \\
\hline 41 & $\begin{array}{c}\text { Herrero R, Castellsagué X, Pawlita M, Lissowska J, Kee F, Balaram P, Rajkumar } \\
\text { T, Sridhar H, Rose B, Pintos J, Fernández L, Idris A, Sánchez MJ, Nieto A, Talamini } \\
\text { R, Tavani A, Bosch FX, Reidel U, Snijders PJ, Meijer CJ, Viscidi R, Muñoz N, Franceschi } \\
\text { S; IARC Multicenter Oral Cancer Study Group. Human papillomavirus and oral } \\
\text { cancer: the International Agency for Research on Cancer multicenter study. J Natl } \\
\text { Cancer Inst. 2003;95(23):1772-83. }\end{array}$ & 819 & 51.19 & 854 & 23 \\
\hline 42 & Fox RI. Siögren's syndrome. Lancet. 2005;366(9482):321-31. & 816 & 58.29 & 869 & 6 \\
\hline 43 & $\begin{array}{c}\text { Nutting CM, Morden JP, Harrington KJ, Urbano TG, Bhide SA, Clark C, Miles } \\
\text { EA, Miah AB, Newbold K, Tanay M, Adab F, Jefferies SJ, Scrase C, Yap BK, A'Hern } \\
\text { RP, Sydenham MA, Emson M, Hall E; PARSPORT trial management group. Parotid- } \\
\text { sparing intensity modulated versus conventional radiotherapy in head and neck } \\
\text { cancer (PARSPORT): a phase } 3 \text { multicentre randomised controlled trial. Lancet Oncol. } \\
2011 ; 12(2): 127-36 .\end{array}$ & 810 & 101.25 & 912 & 21 \\
\hline 44 & $\begin{array}{c}\text { Calais G, Alfonsi M, Bardet E, Sire C, Germain T, Bergerot P, Rhein B, Tortochaux } \\
\text { J, Oudinot P, Bertrand P. Randomized trial of radiation therapy versus concomitant } \\
\text { chemotherapy and radiation therapy for advanced-stage oropharynx carcinoma. J Natl } \\
\text { Cancer Inst. } 1999 ; 91(24): 2081-6 \text {. }\end{array}$ & 801 & 40.05 & 878 & - \\
\hline 45 & $\begin{array}{l}\text { Kim JW, Dang CV. Cancer's molecular sweet tooth and the Warburg effect. Cancer } \\
\qquad \text { Res. 2006;66(18):8927-30. }\end{array}$ & 795 & 61.15 & 801 & 75 \\
\hline 46 & $\begin{array}{l}\text { Bernier J, Cooper JS, Pajak TF, van Glabbeke M, Bourhis J, Forastiere A, Ozsahin } \\
\text { EM, Jacobs JR, Jassem J, Ang KK, Lefèbvre JL. Defining risk levels in locally advanced } \\
\text { head and neck cancers: a comparative analysis of concurrent postoperative radiation } \\
\text { plus chemotherapy trials of the EORTC (\#22931) and RTOG (\# 9501). Head Neck. } \\
\qquad 2005 ; 27(10): 843-50 .\end{array}$ & 789 & 56.36 & 927 & 16 \\
\hline 47 & $\begin{array}{c}\text { Bittle JL, Houghten RA, Alexander H, Shinnick TM, Sutcliffe JG, Lerner RA, Rowlands } \\
\text { DJ, Brown F. Protection against foot-and-mouth disease by immunization with a } \\
\text { chemically synthesized peptide predicted from the viral nucleotide sequence. Nature. } \\
\qquad 1982 ; 298(5869): 30-3 .\end{array}$ & 786 & 21.24 & 685 & 9 \\
\hline 48 & $\begin{array}{l}\text { Klein RS, Harris CA, Small CB, Moll B, Lesser M, Friedland GH. Oral candidiasis } \\
\text { in high-risk patients as the initial manifestation of the acquired immunodeficiency } \\
\text { syndrome. N Engl J Med. 1984;311(6):354-8. }\end{array}$ & 768 & 21.94 & 709 & 9 \\
\hline 49 & $\begin{array}{l}\text { Foucar E, Rosai J, Dorfman R. Sinus histiocytosis with massive lymphadenopathy } \\
\text { (Rosai-Dorfman disease): review of the entity. Semin Diagn Pathol. 1990;7(1):19-73. }\end{array}$ & 767 & 26.45 & 773 & 3 \\
\hline 50 & $\begin{array}{l}\text { Bamias A, Kastritis E, Bamia C, Moulopoulos LA, Melakopoulos I, Bozas G, } \\
\text { Koutsoukou V, Gika D, Anagnostopoulos A, Papadimitriou C, Terpos E, Dimopoulos } \\
\text { MA. Osteonecrosis of the jaw in cancer after treatment with bisphosphonates: } \\
\text { incidence and risk factors. J Clin Oncol. } 2005 ; 23(34): 8580-7 \text {. }\end{array}$ & 763 & 54.50 & 814 & 6 \\
\hline 51 & Grubman MJ, Baxt B. Foot-and-mouth disease. Clin Microbiol Rev. 2004;17(2):465-93. & 763 & 50.87 & 692 & 13 \\
\hline
\end{tabular}


Acharya R, Fry E, Stuart D, Fox G, Rowlands D, Brown F. The three-dimensional

761

25.37

684

Hong WK, Endicott J, Itri LM, Doos W, Batsakis JG, Bell R, Fofonoff S, Byers $R$, Atkinson EN, Vaughan C, et al. 13-cis-retinoic acid in the treatment of oral leukoplakia. N Engl J Med. 1986;315(24):1501-5.

Groom J, Kalled SL, Cutler AH, Olson C, Woodcock SA, Schneider P, Tschopp J, Cachero TG, Batten M, Wheway J, Mauri D, Cavill D, Gordon TP, Mackay CR, Mackay F. Association of BAFF/BLyS overexpression and altered B cell differentiation with Siögren's syndrome. J Clin Invest. 2002;109(1):59-68.

Proetzel G, Pawlowski SA, Wiles MV, Yin M, Boivin GP, Howles PN,

Ding J, Ferguson MW, Doetschman T. Transforming growth factor-beta

3 is required for secondary palate fusion. Nat Genet. 1995; 1 1 (4):409-14.

Silverman S Jr, Gorsky M, Lozada F. Oral leukoplakia and malignant transformation. A follow-up study of 257 patients. Cancer. 1984;53(3):563-8.

Dixon MJ, Marazita ML, Beaty TH, Murray JC. Cleft lip and palate: understanding genetic and environmental influences. Nat Rev Genet. $2011 ; 12(3): 167-78$.

Semina EV, Reiter R, Leysens NJ, Alward WL, Small KW, Datson NA, Siegel-

Bartelt J, Bierke-Nelson D, Bitoun P, Zabel BU, Carey JC, Murray JC. Cloning and characterization of a novel bicoid-related homeobox transcription factor gene, RIEG, involved in Rieger syndrome. Nat Genet. 1996; 14(4):392-9.

59 Isaacson PG, Spencer J. Malignant lymphoma of mucosa-associated lymphoid tissue. Histopathology. 1987; 1 1 (5):445-62.

Rheinwald JG, Beckett MA. Tumorigenic keratinocyte lines requiring anchorage and fibroblast support cultured from human squamous cell carcinomas. Cancer Res. 1981 ; 4 (5):1657-63.

Colella G, Campisi G, Fusco V. American Association of Oral and Maxillofacial Surgeons

61 position paper: Bisphosphonate-Related Osteonecrosis of the Jaws-2009 update: the need to refine the BRONJ definition. J Oral Maxillofac Surg. 2009;67(12):2698-9.

Ruggiero SL, Dodson TB, Fantasia J, Goodday R, Aghaloo T, Mehrotra B, O’Ryan

F; American Association of Oral and Maxillofacial Surgeons. American Association of

Oral and Maxillofacial Surgeons position paper on medication-related osteonecrosis of the jaw--2014 update. J Oral Maxillofac Surg. 2014;72(10):1938-56.

Corrao G, Bagnardi V, Zambon A, La Vecchia C. A meta-analysis of alcohol consumption and the risk of 15 diseases. Prev Med. 2004;38(5):613-9.

Greenspan JS, Greenspan D, Lennette ET, Abrams DI, Conant MA, Petersen V, Freese UK. Replication of Epstein-Barr virus within the epithelial cells of oral "hairy" leukoplakia, an AIDS-associated lesion. N Engl J Med. 1985;313(25):1564-71. Neville BW, Day TA. Oral cancer and precancerous lesions. CA Cancer J Clin. 2002;52(4):195-215.

Eisbruch A, Ten Haken RK, Kim HM, Marsh LH, Ship JA. Dose, volume, and function 66 relationships in parotid salivary glands following conformal and intensity-modulated irradiation of head and neck cancer. Int J Radiat Oncol Biol Phys. 1999;45(3):577-87.

Trotti A, Bellm LA, Epstein JB, Frame D, Fuchs HJ, Gwede CK, Komaroff E, Nalysnyk

$67 \mathrm{~L}$, Zilberberg MD. Mucositis incidence, severity and associated outcomes in patients with head and neck cancer receiving radiotherapy with or without chemotherapy: a systematic literature review. Radiother Oncol. 2003;66(3):253-62.

68 Lindberg R. Distribution of cervical lymph node metastases from squamous cell carcinoma of the upper respiratory and digestive tracts. Cancer. 1972;29(6):1446-9.

69 Foote FW, Frazell EL. Tumors of the major salivary glands. Cancer. 1953;6(6):1065-133.

Stothard KJ, Tennant PW, Bell R, Rankin J. Maternal overweight and obesity and

70 the risk of congenital anomalies: a systematic review and meta-analysis. JAMA. 2009;301 (6):636-50.

71 Skre H. Genetic and clinical aspects of Charcot-Marie-Tooth's disease. Clin Genet. 1974;6(2):98-118.

72 Mossey PA, Little J, Munger RG, Dixon MJ, Shaw WC. Cleft lip and palate. Lancet. 2009;374(9703):1773-85. $\begin{array}{llll}713 & 21.61 \quad 629 \quad 6\end{array}$

$\begin{array}{llll}712 & 41.88 & 692 & 9\end{array}$

$\begin{array}{lll}705 & 29.38 & 691\end{array}$

$\begin{array}{llll}700 & 20.00 \quad 357 \quad 3\end{array}$

$696 \quad 87.00 \quad 759 \quad 11$

$\begin{array}{llll}681 & 29.61 & 695 & 6\end{array}$

$\begin{array}{lll}676 & 21.13 \quad 689\end{array}$

$\begin{array}{lll}665 & 17.50 \quad 535-\end{array}$

$663 \quad 66.30 \quad 66$

$\begin{array}{llll}657 & 131.40 & 833 & 28\end{array}$

$\begin{array}{llll}653 & 43.53 & 675 & 199\end{array}$

$\begin{array}{llll}644 & 18.94 & 685 & 3\end{array}$

$\begin{array}{llll}641 & 37.71 & 675 & 7\end{array}$

$642 \quad 32.10 \quad 709$

$641 \quad 40.06 \quad 670$

$\begin{array}{llll}632 & 13.45 & 768 & 7 \\ 628 & 9.52 & 895 & - \\ 626 & 62.60 & 726 & 22 \\ 618 & 13.73 & 604 & 3 \\ 614 & 61.40 & 663 & 8\end{array}$

Continue... 
Mork J, Lie AK, Glattre E, Hallmans G, Jellum E, Koskela P, Møller B, Pukkala $E$, Schiller JT, Youngman L, Lehtinen M, Dillner J. Human papillomavirus infection as a risk factor for squamous-cell carcinoma of the head and neck. N Engl J Med. $2001 ; 344(15): 1125-31$.

Chan G, Boyle JO, Yang EK, Zhang F, Sacks PG, Shah JP, Edelstein D, Soslow RA, Koki AT, Woerner BM, Masferrer JL, Dannenberg AJ. Cyclooxygenase-2 expression is up-regulated in squamous cell carcinoma of the head and neck. Cancer Res. 1999;59(5):991-4.

Grandis JR, Tweardy DJ. Elevated levels of transforming growth factor alpha and 75 epidermal growth factor receptor messenger RNA are early markers of carcinogenesis in head and neck cancer. Cancer Res. 1993;53(15):3579-84.

Rehm J, Room R, Graham K, Monteiro M, Gmel G, Sempos CT. The relationship of

76 average volume of alcohol consumption and patterns of drinking to burden of disease: an overview. Addiction. 2003;98(9):1209-28.

Soulieres D, Senzer NN, Vokes EE, Hidalgo M, Agarwala SS, Siu LL. Multicenter phase II study of erlotinib, an oral epidermal growth factor receptor tyrosine kinase inhibitor, in patients with recurrent or metastatic squamous cell cancer of the head and neck. J Clin Oncol. 2004;22(1):77-85.

Shimizu T, Ehrlich GE, Inaba G, Hayashi K. Behçet disease (Behçet syndrome). Semin Arthritis Rheum. 1979;8(4):223-60.

Greenspan JS, Daniels TE, Talal N, Sylvester RA. The histopathology of Siögren's syndrome in labial salivary gland biopsies. Oral Surg Oral Med Oral Pathol. 1974;37(2):217-29.

Brennan JA, Mao L, Hruban RH, Boyle JO, Eby YJ, Koch WM, Goodman

$80 \mathrm{SN}$, Sidransky D. Molecular assessment of histopathological staging in squamous-cell carcinoma of the head and neck. N Engl J Med. 1995;332(7):429-35.

Warnakulasuriya S, Johnson NW, van der Waal I. Nomenclature and classification of potentially malignant disorders of the oral mucosa. J Oral Pathol Med. 2007;36(10):575-80.

Chajek T, Fainaru M. Behçet's disease. Report of 41 cases and a review of the literature. Medicine (Baltimore). 1975;54(3): 179-96.

Denis F, Garaud P, Bardet E, Alfonsi M, Sire C, Germain T, Bergerot P, Rhein B, Tortochaux

83 J, Calais G. Final results of the 94-01 French Head and Neck Oncology and Radiotherapy

Group randomized trial comparing radiotherapy alone with concomitant radiochemotherapy in advanced-stage oropharynx carcinoma. J Clin Oncol. 2004;22(1):69-76.

Conti HR, Shen F, Nayyar N, Stocum E, Sun JN, Lindemann MJ, Ho AW, Hai JH, Yu JJ, Jung JW, Filler SG, Masso-Welch P, Edgerton M, Gaffen SL. Th17 cells and IL-17 receptor signaling are essential for mucosal host defense against oral candidiasis. J Exp Med. 2009;206(2):299-31 1

Wong TS, Liu XB, Wong BY, Ng RW, Yuen AP, Wei WI. Mature miR-184 as Potential 85 Oncogenic microRNA of Squamous Cell Carcinoma of Tongue. Clin Cancer Res. 2008; 14(9):2588-92.

van den Brekel MW, Stel HV, Castelijns JA, Nauta JJ, van der Waal I, Valk J, Meyer

86 CJ, Snow GB. Cervical lymph node metastasis: assessment of radiologic criteria. Radiology. 1990;177(2):379-84.

Sullivan DJ, Westerneng TJ, Haynes KA, Bennett DE, Coleman DC. Candida dubliniensis

87 sp. nov.: phenotypic and molecular characterization of a novel species associated with oral candidosis in HIV-infected individuals. Microbiology. 1995; 141 (P+ 7):1507-21.

Puel A, Cypowyi S, Bustamante J, Wright JF, Liu L, Lim HK, Migaud M, Israel

L, Chrabieh M, Audry M, Gumbleton M, Toulon A, Bodemer C, El-Baghdadi

88 J, Whitters M, Paradis T, Brooks J, Collins M, Wolfman NM, Al-Muhsen S, Galicchio

M, Abel L, Picard C, Casanova JL. Chronic mucocutaneous candidiasis in humans with inborn errors of interleukin-17 immunity. Science. 2011 ;332(6025):65-8.
$611 \quad 33.94 \quad 632 \quad 8$

$610 \quad 30.50 \quad 477$

$607 \quad 23.35 \quad 534 \quad 7$

$605 \quad 37.81 \quad 642 \quad 32$

$603 \quad 40.20 \quad 628 \quad 6$

$596 \quad 14.90 \quad 484$

$595 \quad 13.22 \quad 513$

$594 \quad 24.75 \quad 570 \quad 3$

$593 \quad 49.42 \quad 702 \quad 5$

$585 \quad 13.30 \quad 506$

$580 \quad 38.67 \quad 640$

$\begin{array}{llll}577 & 57.70 & 612 & 8\end{array}$

$\begin{array}{llll}573 & 52.09 & 547 & 12\end{array}$

$573 \quad 19.76 \quad 563$

$572 \quad 23.83 \quad 549$

12

$\begin{array}{llll}568 & 71.00 \quad 640 \quad 18\end{array}$

Weinberger PM, Yu Z, Haffty BG, Kowalski D, Harigopal M, Brandsma J, Sasaki C, Joe

J, Camp RL, Rimm DL, Psyrri A. Molecular classification identifies a subset of human papillomavirus--associated oropharyngeal cancers with favorable prognosis. J Clin Oncol. 2006;24(5):736-47.

$\begin{array}{llll}556 & 42.77 \quad 588 & 6\end{array}$


...Continuation

Forastiere AA, Metch B, Schuller DE, Ensley JF, Hutchins LF, Triozzi P, Kish JA, McClure

$S$, Vonfeldt E, Williamson SK, et al. Randomized comparison of cisplatin plus

90 fluorouracil and carboplatin plus fluorouracil versus methotrexate in advanced squamous-cell carcinoma of the head and neck: a Southwest Oncology Group study. J Clin Oncol. 1992;10(8):1245-51.

91 Sonis ST. The pathobiology of mucositis. Nat Rev Cancer. 2004;4(4):277-84.

92 Dahlin DC, Coventry MB. Osteogenic sarcoma. A study of six hundred cases. J Bone Joint Surg Am. 1967;49(1):101-10.

$556 \quad 20.59 \quad 535$

$555 \quad 37.00 \quad 590 \quad 3$

$551 \quad 10.60 \quad 471$

550

$78.57 \quad 620$

143

Gillison ML, Broutian T, Pickard RK, Tong ZY, Xiao W, Kahle L, Graubard

$93 \mathrm{BI}$, Chaturvedi AK. Prevalence of oral HPV infection in the United States, 2009-2010. JAMA. 2012;307(7):693-703.

94 Isaacson P, Wright DH. Extranodal malignant lymphoma arising from mucosaassociated lymphoid tissue. Cancer. 1984;53(1 1):2515-24.

550

15.71

609

Brizel DM, Wasserman TH, Henke M, Strnad V, Rudat V, Monnier A, Eschwege

95 F, Zhang J, Russell L, Oster W, Sauer R. Phase III randomized trial of amifostine as a radioprotector in head and neck cancer. J Clin Oncol. 2000;18(19):3339-45.

Advisory Task Force on Bisphosphonate-Related Ostenonecrosis of the Jaws, American Association of Oral and Maxillofacial Surgeons. American Association of Oral and Maxillofacial Surgeons position paper on bisphosphonaterelated osteonecrosis of the jaws. J Oral Maxillofac Surg. 2007;65(3):369-76.

45.58

658

622

Gorlin RJ. Nevoid basal-cell carcinoma syndrome. Medicine (Baltimore). 1987;66(2):98-113.

547

17.09

626

Satokata I, Ma L, Ohshima H, Bei M, Woo I, Nishizawa K, Maeda T, Takano Y, Uchiyama M, Heaney S, Peters H, Tang Z, Maxson R, Maas R. Msx2 deficiency in mice causes pleiotropic defects in bone growth and ectodermal organ formation. Nat Genet. 2000;24(4):391-5.

Zhao C, Takita J, Tanaka Y, Setou M, Nakagawa T, Takeda S, Yang HW, Terada 99 S, Nakata T, Takei Y, Saito M, Tsuji S, Hayashi Y, Hirokawa N. Charcot-MarieTooth disease type $2 \mathrm{~A}$ caused by mutation in a microtubule motor KIF1Bbeta. Cell. 2001;105(5):587-97.

Hashibe M, Brennan P, Benhamou S, Castellsague X, Chen C, Curado MP, Dal Maso L, Daudt AW, Fabianova E, Fernandez L, Wünsch-Filho V, Franceschi S, Hayes RB, Herrero

R, Koifman S, La Vecchia C, Lazarus P, Levi F, Mates D, Matos E, Menezes A, Muscat J, ElufNeto J, Olshan AF, Rudnai P, Schwartz SM, Smith E, Sturgis EM, Szeszenia-Dabrowska

100 N, Talamini R, Wei Q, Winn DM, Zaridze D, Zatonski W, Zhang ZF, Berthiller J, Boffetta P. Alcohol drinking in never users of tobacco, cigarette smoking in never drinkers, and the risk of head and neck cancer: pooled analysis in the International Head and Neck Cancer Epidemiology Consortium. J Natl Cancer Inst. 2007;99(10):777-89.

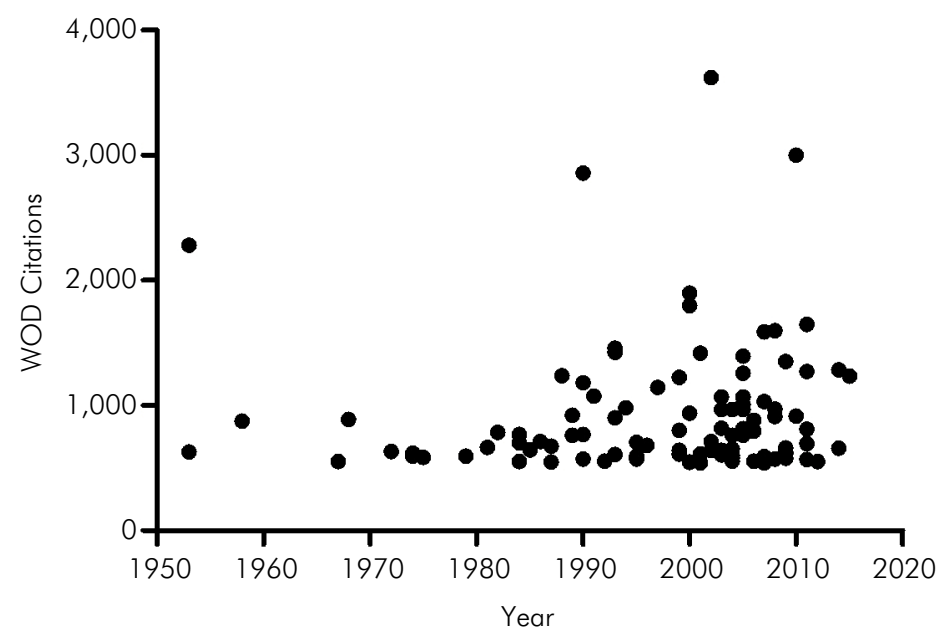

Figure 1. Number of citations per year. 
different journals, with $89 \%$ published in non-specific journals for oral medicine and pathology. The New England Journal of Medicine was the journal with the highest number of papers in the top-100 followed by the American Cancer Society and Nature Genetics, accounting for 24 papers out of 100 (Table 2).

The authors belonged to institutions in 18 different countries. Most were from institutions in the United States (55\%) and Europe (35\%). Papers were published in both developed (e.g., Japan, Australia, Canada, and Israel) and developing countries (e.g., China, Costa Rica, Taiwan, and Uganda) (Table 3). The most frequent field of study was head and neck cancer $(n=38)$, followed by congenital craniofacial anomaly $(n=8)$ and osteonecrosis $(\mathrm{n}=8)$ (Table 4). The most common methodological designs were laboratory and descriptive studies, which accounted for $59 \%$ of the papers. Few studies have been clinical, retrospective, or comparative studies, or case-reports (Table 5).

In the correlation analysis, a strong correlation was found between the number of citations in WOS and the total number of citations in Dimensions $(r=0.976$, $\mathrm{p}<0.0001)$. A clear linear correlation between the number of citations of the papers in both databases can be seen in the scatter plot graph in Figure 2. Furthermore, a poor correlation was found between AAS and the number of citations in WOS for those papers which had an AAS $(r=0.147, p=0.185)$.

\section{Discussion}

Analyzing the most frequently cited papers in a specific area enables researchers to identify the evolution of research in that area over time and to gain a better understanding of the areas of most interest and the existing gaps. ${ }^{1,2}$ We aimed to identify and analyze the 100 most-cited papers in the field of oral medicine and pathology. Oral medicine and pathology are specialties at the intersection of medicine and dentistry, focused on oral symptoms, diagnosis, and the oral care of medically compromised patients. ${ }^{21}$ These fields were identified as specialties by Dr. Samuel Charles Miller when he founded the American Academy of Oral Medicine in 1945 (https://www.aaom.com/history).
However, because oral medicine and pathology were considered medical fields for many years, there are few consolidated journals in our area and many researchers choose to submit to a medical journal. This is one possible explanation for why most of the publications were in non-specific journals. Of the 47 journals in which the top-100 cited papers were published, only 6 were publications specifically on oral medicine and pathology.

A citation classic refers to a paper with 100 or more citations; however, this number varies according to specialty. $8,9,10,11,12,13,14,15,16,17,18,19$ In this study, the 100 most-cited papers in oral medicine and pathology received a greater number of citations, ranging from 541 to 3623 . Therefore, all the papers on our list of the 100 most-cited are classics, as in some studies in dentistry. ${ }^{11,12,13,14,15,18,19}$ However, other bibliometric studies in dentistry have listed papers with less than 100 citations. ${ }^{8,9,10,16,17,20}$ This discrepancy is possible because in addition to being a very specific area of dentistry, some journals were non-specific in terms of the area studied, as in our study.

In oral medicine and pathology, head and neck cancer is the most worrying disease, and numerous studies have been conducted on this topic over time. ${ }^{22}$ Therefore, studies targeted at understanding, preventing, and/or treating head and neck cancer have made this condition the most-cited thematic field in the 100 most-cited papers in oral medicine and pathology. Congenital craniofacial anomaly and osteonecrosis were the others fields most-cited in the top 100 list, reflecting the holistic manner in which oral medicine and pathology deals with health.

Although the numbers of reviews are rapidly increasing in dentistry, ${ }^{23}$ only $9 \%$ of the 100 mostcited oral medicine and pathology papers were reviews (systematic reviews $4 \%$, literature reviews $3 \%$, and meta-analyses $2 \%$ ). Thus, as authors prefer to cite reviews over research with a lower level of evidence, ${ }^{24}$ additional systematic reviews and metaanalyses are needed for various focused questions in oral medicine and pathology. Fifty nine percent of the papers were laboratory and descriptive studies. This finding is not surprising since these types of studies are the precursors and subsequent studies cite them. 
The 100 most-cited papers in oral medicine and pathology

Table 2. The 47 journals in which the top- 100 cited papers were published.

\begin{tabular}{|c|c|c|c|}
\hline Journal & $\mathrm{N}^{\circ}$ of papers & ISSN & Published since \\
\hline The New England Journal of Medicine & 10 & $0028-4793$ & 1812 \\
\hline American Cancer Society & 7 & $1097-0142$ & 1913 \\
\hline Nature Genetics & 7 & 1546-1718 & 1992 \\
\hline Journal of Oral Maxillofacial Surgery & 6 & $1531-5053$ & 1943 \\
\hline Journal of the National Cancer Institute & 6 & $1460-2105$ & 1940 \\
\hline The Lancet & 6 & $1474-547 X$ & 1823 \\
\hline Cancer Research & 5 & $1538-7445$ & 1916 \\
\hline Journal of Clinical Oncology & 5 & $1527-7755$ & 1983 \\
\hline Cell & 3 & $1097-4172$ & 1974 \\
\hline Nature & 3 & $1476-4687$ & 1869 \\
\hline JAMA & 2 & $1538-3598$ & 1883 \\
\hline Journal of Bone and Mineral Research & 2 & $1535-1386$ & 1889 \\
\hline Journal of Clinical of Oncology & 2 & $1527-7755$ & 1983 \\
\hline Nature Reviews Cancer & 2 & $1474-1768$ & 1869 \\
\hline Science & 2 & 1095- 9203 & 1880 \\
\hline Annals of Internal Medicine & 1 & $1539-3704$ & 1927 \\
\hline Annals of the Rheumatic Diseases & 1 & $1468-2060$ & 1939 \\
\hline Anticancer Research & 1 & $1791-7530$ & 1981 \\
\hline Arthritis e Rheumatology & 1 & $2326-5205$ & 1958 \\
\hline Bull World Health Organ & 1 & $1564-0604$ & 1947 \\
\hline Cancer Epidemiology Biomarkers and Prevention & 1 & $1538-7755$ & 1991 \\
\hline Clinical Cancer Research & 1 & $1557-3265$ & 1995 \\
\hline Clinical Genetics & 1 & 1399-0004 & 1970 \\
\hline Clinical Microbiology & 1 & $1098-660 x$ & 1975 \\
\hline Community Dentistry Oral Epidemiology & 1 & $1600-0528$ & 1973 \\
\hline International Journal of Radiation Oncology & 1 & $0360-3016$ & 1975 \\
\hline Journal of Biological Chemistry & 1 & $1083-351 x$ & 1905 \\
\hline Journal of Clinical Pathology & 1 & $1472-4146$ & 1947 \\
\hline Journal of Community Health & 1 & $1573-3610$ & 1975 \\
\hline Journal of Experimental Medicine & 1 & $1540-9538$ & 1986 \\
\hline Journal of Oral Pathology of Medicine & 1 & $1600-0714$ & 1943 \\
\hline Journal of the Sciences and Specialties of the Head and Neck & 1 & $1097-0347$ & 1995 \\
\hline Medicine (Baltimore) & 1 & $1536-5964$ & 1922 \\
\hline Microbiology & 1 & $1465-2080$ & 1947 \\
\hline Nano Letters & 1 & $1530-6992$ & 2001 \\
\hline Nature Reviews Genetics & 1 & $1471-0064$ & 2000 \\
\hline Oral Oncology & 1 & $1368-8375$ & 1965 \\
\hline Oral Surgery Oral Medicine Oral Pathology & 1 & $2212-4411$ & 1948 \\
\hline Preventive medicine & 1 & 1096-0260 & 1972 \\
\hline Radiology & 1 & 0033-8419 & 1923 \\
\hline
\end{tabular}




\begin{tabular}{llll}
...Continuation & & $0167-8140$ & 1983 \\
\hline Radiotherapy \& Oncology & 1 & $0740-2570$ & $0049-0172$ \\
Seminars in Diagnostic Pathology & 1 & $1360-0443$ & 1971 \\
Seminars in Arthritis and Rheumatism & 1 & 1884 \\
Society for the Study of Addiction & 1 & $1558-8238$ & 1913 \\
The British Journal of Surgery & 1 & $1470-2045$ & 2000 \\
The Journal of Clinical Investigation & 1 & 1323 \\
The Lancet Oncology &
\end{tabular}

Table 3. The 18 countries of origin of the top- 100 cited papers in pathology and oral medicine.

\begin{tabular}{|c|c|c|c|}
\hline \multirow{2}{*}{ Country } & \multirow{2}{*}{ No. of papers } & \multicolumn{2}{|c|}{ Citations } \\
\hline & & WOS & Citations/papers \\
\hline United States of American & 55 & 49777 & 905.03 \\
\hline United Kingdom & 11 & 8160 & 741.81 \\
\hline France & 7 & 8405 & 1200.71 \\
\hline Italy & 4 & 6363 & 1590.75 \\
\hline Switzerland & 4 & 3530 & 882.50 \\
\hline Germany & 3 & 3082 & 1027.33 \\
\hline Japan & 3 & 2363 & 787.67 \\
\hline Netherlands & 2 & 1845 & 992.50 \\
\hline Norway & 2 & 1229 & 614.50 \\
\hline Australia & 1 & 712 & 712.00 \\
\hline Canada & 1 & 603 & 603.00 \\
\hline China & 1 & 573 & 573.00 \\
\hline Costa Rica & 1 & 819 & 819.00 \\
\hline Greece & 1 & 763 & 763.00 \\
\hline Ireland & 1 & 572 & 572.00 \\
\hline Israel & 1 & 585 & 585.00 \\
\hline Taiwan & 1 & 1419 & 1419.00 \\
\hline Uganda & 1 & 874 & 874.00 \\
\hline
\end{tabular}

The vast majority of the most-cited publications (90\%) were from the United States or Europe. This result probably reflects the better funding policies and the large number of researchers that are in these countries. The United States and European countries are also the leading countries in terms of medical research publications. In addition, the citations originate more frequently from institutions located in the same country, ${ }^{25}$ and researchers from different parts of the world often move to these countries (https://www.iom.int/wmr/).
A strong correlation was observed between WOS and Dimensions citation counts. This finding indicates that the new database (Dimensions) can be used as an alternative to WOS. This result was previously observed in a study by Garcovich et al. ${ }^{16}$ However, AAS values were not directly comparable to WOS citation counts. Two other studies also found no correlation between AAS and WOS or Scopus citation count. ${ }^{16,26}$ In addition, Thelwall ${ }^{27}$ reported that Dimensions is comparable in coverage to Scopus, suggesting that WOS, Dimensions and 
The 100 most-cited papers in oral medicine and pathology

Table 4. Study subjects in the 100-top cited papers in pathology and oral medicine.

\begin{tabular}{|c|c|c|c|c|c|}
\hline \multirow{2}{*}{ Study subject } & \multirow{2}{*}{ No. of papers } & \multicolumn{3}{|c|}{ Citations } & \multirow{2}{*}{ AAS } \\
\hline & & WOS & WOS/years & Dimensions & \\
\hline Head and Neck Cancer & 38 & 37646 & 52.43 & 40763 & 2262 \\
\hline Craniofacial congenital anomaly & 8 & 6282 & 47.59 & 6644 & 77 \\
\hline Osteonecrosis & 8 & 6839 & 80.45 & 6915 & 92 \\
\hline Behçet's syndrome & 7 & 7575 & 35.23 & 7151 & 41 \\
\hline Etiology of oral diseases & 6 & 4485 & 56.06 & 4899 & 412 \\
\hline Sjogren's syndrome & 6 & 8059 & 47.41 & 7514 & 35 \\
\hline Pre-malignant lesions & 5 & 4069 & 30.82 & 4073 & 87 \\
\hline Charcot-Marie-Tooth syndrome & 5 & 4105 & 31.09 & 4138 & 40 \\
\hline Oral candidiasis & 4 & 2485 & 32.27 & 2510 & 47 \\
\hline Oral mucositis & 2 & 1196 & 38.58 & 1260 & 3 \\
\hline Salivary gland tumors & 2 & 1551 & 16.15 & 1559 & - \\
\hline MALT* lymphoma & 2 & 1226 & 18.30 & 1298 & - \\
\hline Burkitt's lymphoma & 1 & 874 & 14.33 & 919 & 21 \\
\hline Rosai-Dorfman diasease & 1 & 767 & 26.45 & 773 & 3 \\
\hline Chemotherapy in head and neck cancer & 1 & 1798 & 94.63 & 1900 & 6 \\
\hline Hypophosphataemic rickets & 1 & 938 & 49.36 & 1000 & 6 \\
\hline Osteogenic sarcoma & 1 & 551 & 10.59 & 471 & - \\
\hline Gorlin-Goltz syndrome & 1 & 547 & 17.09 & 626 & 3 \\
\hline Rieger syndrome & 1 & 681 & 29.61 & 695 & 6 \\
\hline
\end{tabular}

*Mucosa-associated lymphoid tissue lymphoma.

Table 5. Study designs used in the 100-top cited papers in pathology and oral medicine.

\begin{tabular}{|c|c|c|c|c|c|}
\hline \multirow{2}{*}{ Study design } & \multirow{2}{*}{ No. of papers } & \multicolumn{3}{|c|}{ Citations } & \multirow{2}{*}{ AAS } \\
\hline & & WOS & WOS/paper & Dimensions & \\
\hline Laboratory & 33 & 27152 & 822.78 & 27694 & 580 \\
\hline Descriptive & 26 & 27395 & 1053.65 & 27628 & 424 \\
\hline Randomized & 8 & 6158 & 769.75 & 6216 & 57 \\
\hline Case-control & 5 & 5166 & 1033.20 & 5454 & 660 \\
\hline Epidemiological & 5 & 4389 & 877.80 & 5429 & 959 \\
\hline Longitudinal & 4 & 2679 & 669.75 & 2577 & 9 \\
\hline Systematic review & 4 & 3408 & 852.00 & 3740 & 64 \\
\hline Cross-sectional & 3 & 1878 & 626.00 & 2267 & 10 \\
\hline Literature review & 3 & 2673 & 891.00 & 2675 & 19 \\
\hline Clinical & 2 & 2022 & 1011.00 & 1928 & 76 \\
\hline Retrospective & 2 & 4288 & 2144.00 & 4700 & 62 \\
\hline Meta-analysis & 2 & 2451 & 1225.50 & 2575 & 205 \\
\hline Case report & 2 & 1226 & 613.00 & 1298 & - \\
\hline Comparative & 1 & 789 & 789.00 & 927 & 16 \\
\hline
\end{tabular}




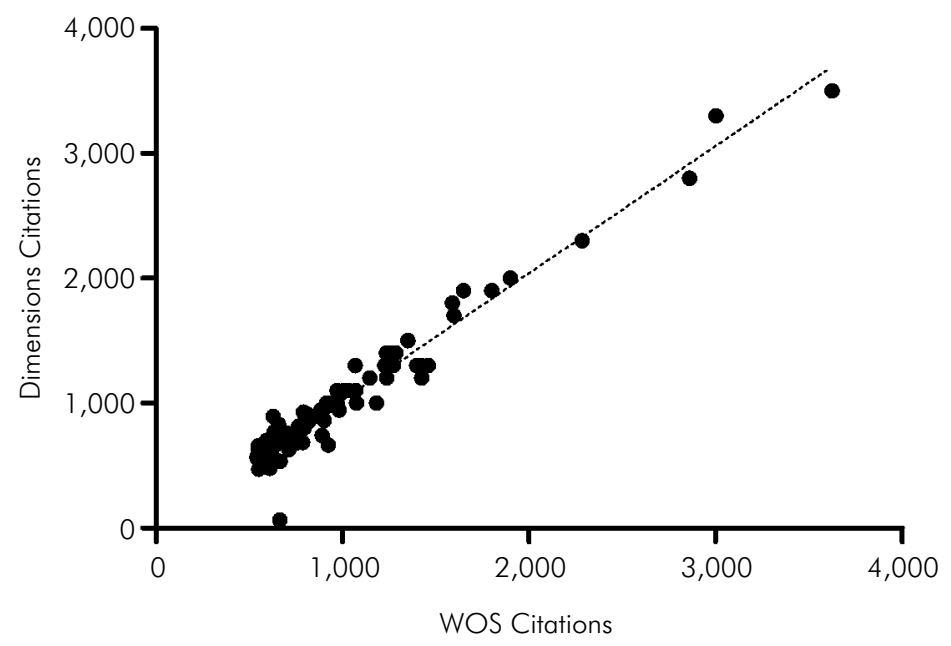

Figure 2. Scatter plot examining the relationship between WOS citations and Dimensions citations.

Scopus can all be used independently to assess research quality.

\section{Conclusion}

In conclusion, this biometric analysis identified the major areas of interest in the field of oral medicine and pathology. The most relevant areas that were explored in the 100 most-cited papers were head and neck cancer, congenital craniofacial anomaly, and osteonecrosis. The majority of the papers were laboratory and descriptive studies and were published in non-specific journals. Together, these findings can help guide the training of oral medicine and pathology students and reveal the gaps in the field.

\section{Acknowledgments}

The authors gratefully acknowledge the Minas Gerais State Research Foundation (Minas Gerais, Brazil), National Council for Scientific and Technological Development (Brasilia, Brazil), and Coordination of Training of Higher Education Graduate Foundation (Brasilia, Brazil) for their assistance and support in carrying out this study.

\section{References}

1. Celeste RK, Broadbent JM, Moyses SJ. Half-century of Dental Public Health research: bibliometric analysis of world scientific trends. Community Dent Oral Epidemiol. 2016 Dec;44(6):557-63. https://doi.org/10.1111/cdoe.12249

2. Aksnes DW, Langfeldt L, Wouters P. Citations, Citation indicators, and research quality: an overview of basic concepts and theories. SAGE Open. 2019;9(1):1-7. https://doi.org/10.1177/2158244019829575

3. Pena-Cristóbal M, Diniz-Freitas M, Monteiro L, Diz Dios P, Warnakulasuriya S. The 100 most cited articles on oral cancer. J Oral Pathol Med. 2018 Apr;47(4):333-44. https://doi.org/10.1111/jop.12686

4. Garfield E. 100 citation classics from the Journal of the American Medical Association. JAMA. 1987 Jan;257(1):52-9. https://doi.org/10.1001/jama.1987.03390010056028

5. Lucena MF, Teixeira PE, Bonin Pinto C, Fregni F. Top 100 cited noninvasive neuromodulation clinical trials. Expert Rev Med Devices. 2019 Jun;16(6):451-66. https://doi.org/10.1080/17434440.2019.1615440

6. Montinaro V, Giliberti M, Villani C, Montinaro A. Citation classics: ranking of the top 100 most cited articles in nephrology. Clin Kidney J. 2019 Feb;12(1):6-18. https://doi.org/10.1093/cki/sfy033

7. Xie L, Chen Z, Wang H, Zheng C, Jiang J. Bibliometric and visualized analysis of scientific publications on atlantoaxial spine surgery based on Web of Science and VOSviewer. World Neurosurg. 2020 May;137(May):435-442.e4. https://doi.org/10.1016/i.wneu.2020.01.171 
The 100 most-cited papers in oral medicine and pathology

8. Jafarzadeh H, Sarraf Shirazi A, Andersson L. The most-cited articles in dental, oral, and maxillofacial traumatology during 64 years. Dent Traumatol. 2015 Oct;31(5):350-60. https://doi.org/10.1111/edt.12195

9. Fardi A, Kodonas K, Gogos C, Economides N. Top-cited articles in endodontic journals. J Endod. 2011 Sep;37(9):1183-90. https://doi.org/10.1016/i.joen.2011.05.037

10. Adnan S, Ullah R. Top-cited papers in regenerative endodontics: a bibliometric analysis. J Endod. 2018 Nov;44(11):1650-64. https://doi.org/10.1016/i.joen.2018.07.015

11. Yılmaz B, Dinçol ME, Yalçın TY. A bibliometric analysis of the 103 top-cited articles in endodontics. Acta Odontol Scand. 2019 Nov;77(8):574-83. https://doi.org/10.1080/00016357.2019.1621378

12. Feijoo JF, Limeres J, Fernández-Varela M, Ramos I, Diz P. The 100 most cited articles in dentistry. Clin Oral Investig. 2014 Apr;18(3):699-706. https://doi.org/10.1007/s00784-013-1017-0

13. Alarcón MA, Esparza D, Montoya C, Monje A, Faggion CM Jr. The 300 most-cited papers in implant dentistry. Int J Oral Maxillofac Implants. 2017 Jan/Feb;32(1):el-8. https://doi.org/10.11607/jomi.5104

14. Chiang HS, Huang RY, Weng PW, Mau LP, Su CC, Tsai YC, et al. Increasing prominence of implantology research: a chronological trend analysis of 100 top-cited articles in periodontal journals. Eur J Oral Implantol. 2018;11(1):97-110.

15. Fardi A, Kodonas K, Lillis T, Veis A. Top-cited papers in implant dentistry. Int J Oral Maxillofac Implants. 2017 May/Jun;32(3):555-64. https://doi.org/10.11607/jomi.5331

16. Garcovich D, Marques Martinez L, Adobes Martin M. Citation classics in paediatric dentistry: a bibliometric study on the 100 most-cited articles. Eur Arch Paediatr Dent. 2020 Apr;21(2):249-61. https://doi.org/10.1007/s40368-019-00483-z

17. Perazzo MF, Otoni AL, Costa MS, Granville-Granville AF, Paiva SM, Martins-Júnior PA. The top 100 most-cited papers in Paediatric Dentistry journals: A bibliometric analysis. Int J Paediatr Dent. 2019 Nov;29(6):692-711. https://doi.org/10.1111/ipd.12563

18. Faggion CM Jr, Málaga L, Monje A, Trescher AL, Listl S, Alarcón MA. The 300 most cited articles published in periodontology. Clin Oral Investig. 2017 Jul;21(6):2021-8. https://doi.org/10.1007/s00784-016-1990-1

19. Tarazona B, Lucas-Dominguez R, Paredes-Gallardo V, Alonso-Arroyo A, Vidal-Infer A. The 100 most-cited articles in orthodontics: a bibliometric study. Angle Orthod. 2018 Nov;88(6):785-96. https://doi.org/10.2319/012418-65.1

20. Hui J, Han Z, Geng G, Yan W, Shao P. The 100 top-cited articles in orthodontics from 1975 to 2011. Angle Orthod. 2013 May;83(3):491-9. https://doi.org/10.2319/040512-284.1

21. Spielman Al. The future of oral medicine. Oral Dis. 2018 Mar;24(1-2):285-8. https://doi.org/10.1111/odi.12739

22. Schutte HW, Heutink F, Wellenstein DJ, Broek GB, Hoogen FJ, Marres HA, et al. Impact of time to diagnosis and treatment in head and neck cancer: a systematic review. Otolaryngol Head Neck Surg. 2020 Apr;162(4):446-57. https://doi.org/10.1177/0194599820906387

23. Bassani R, Pereira GK, Page MJ, Tricco AC, Moher D, Sarkis-Onofre R. Systematic reviews in dentistry: current status, epidemiological and reporting characteristics. J Dent. 2019 Mar;82:71-84. https://doi.org/10.1016/i.jdent.2019.01.014

24. Montori VM, Wilczynski NL, Morgan D, Haynes RB; Hedges Team. Systematic reviews: a cross-sectional study of location and citation counts. BMC Med. 2003 Nov; 1(1):2. https://doi.org/10.1186/1741-7015-1-2

25. Pasterkamp G, Rotmans JI, De Kleun DV, Borst C. Citation frequency: a biased measure of research impact significantly influenced by the geographical origin of research papers. Scientometrics. 2007 Jan;70(1):153-65. https://doi.org/10.1007/s11192-007-0109-5

26. Livas C, Delli K. Looking beyond traditional metrics in orthodontics: an Altmetric study on the most discussed papers on the web. Eur J Orthod. 2018 Apr;40(2):193-9. https://doi.org/10.1093/ejo/cjx050 PMID:29016742

27. Thelwall M. Dimension: a competitor to Scopus and Web of science? J Informetrics. 2018 May; 12(2):430-5. https://doi.org/10.1016/j.joi.2018.03.006 